\title{
Percepción de estudiantes frente a procesos de enseñanza- aprendizaje durante pandemia por covid-19
}

\section{Elizabeth Londoño-Velasco}

https://orcid.org/oooo-0002-8137-2169 Pontificia Universidad Javeriana, Cali, Colombia

elivelasco@javerianacali.edu.co

\section{Angélica García}

https://orcid.org/oooo-0oo1-8570-1591 Pontificia Universidad Javeriana, Cali, Colombia

angelica.garcia@javerianacali.edu.co

\section{Diana Marcela Osorio-Roa}

https://orcid.org/0ooo-0002-6405-699X Pontificia Universidad Javeriana, Cali, Colombia

diana.osorio@javerianacali.edu.co

\section{Estefanía Montoya-Cobo}

https://orcid.org/0ooo-0003-4182-392X Pontificia Universidad Javeriana, Cali, Colombia

estefania.montoya@javerianacali.edu.co

\section{Ivon Andrea Bolaños-Martínez}

https://orcid.org/oooo-0002-8007-8929 Pontificia Universidad Javeriana, Cali, Colombia ivon.bolanos@javerianacali.edu.co

\section{Germán Darío Isaza Gómez}

https://orcid.org/oooo-0oo1-8475-9994 Institución Universitaria Escuela Nacional del Deporte, Colombia german.isaza@endeporte.edu.co

\section{Resumen}

La llegada de la pandemia por la covid-19 revolucionó la educación universitaria, modificó las estrategias pedagógicas utilizadas por los maestros y las formas de aprender de los estudiantes. El objetivo del estudio fue describir la percepción de estudiantes de una facultad de Ciencias de la Salud frente a los procesos de enseñanzaaprendizaje durante la pandemia por la covid-19. El estudio mixto de tipo descriptivo de corte transversal estuvo constituido por una muestra no probabilistica por conveniencia de 74 estudiantes; el instrumento utilizado fue la encuesta estructurada. El análisis de la información se realizó a través de estadistica básica descriptiva y análisis del discurso. La mayoría de los estudiantes encuestados afirman que no estaban preparados para la educación virtual; solo una quita parte de la muestra afirmo tener competencias suficientes para el uso avanzado de las herramientas de la virtua-

Recepción: 30/og/2020 | Envío a pares: 18/12/2020 | Aceptación por pares: 18/04/2021 | Aprobación: 09/06/2021 
ISSN 0123-1294 | e-ISSN 2027-5358 | Educ.Educ. Vol. 24. No. 2 | Mayo-julio de 2021 | pp. 199-217

Universidad de La Sabana | Facultad de Educación

lidad. No se encontraron diferencias significativas entre la clase magistral y el aula invertida, pero. no ahondamos en las razones. Tampoco, encontramos diferencias significativas entre los métodos de enseñanza presentes en la población estudiada. Las competencias de aprendizaje que, según la muestra, más se desarrollaron fueron la disciplina y la autonomí; sin embargo, la mayor parte de la población considera que la virtualidad le ha quitado rigor al proceso educativo.

\section{Palabras clave (Fuente: tesauro de la Unesco)}

Aprendizaje; clase magistral; enseñanza; pandemia; TIC. 


\title{
Student's Perception of the Teaching-Learning Process during the COVID-19 Pandemic
}

\begin{abstract}
The outbreak of the COVID-19 pandemic convulsed university education and modified professors' pedagogical strategies and students' ways of learning, forcing a transformation from campus-based education to one mediated by virtual environments. The study aims to describe the perception of students from the Faculty of Health of the teaching-learning processes in flipped classrooms and lectures during the pandemic. The mixed descriptive cross-sectional study consisted of an intentional non-probabilistic sample of 74 students. The instrument used was the structured survey, and the data analysis was carried out using basic descriptive statistics and discourse analysis. The results showed that flipped classrooms further promote selforganization because of asynchronous classes and create opportunities for reflection on their learning through forums and spontaneous class participation. Regarding formative and even summative assessments, students perceived this scenario positively and consider that lectures help achieve both the learning objectives in the study plan and promote meaningful learning. In conclusion, remote education is a valuable strategy to continue university education in times of pandemic; however, students believe that it has stripped the educational process of its rigor.
\end{abstract}

\section{Keywords (Source: Unesco Thesaurus)}

Learning; lecture; teaching; pandemic; information and communications technology; ICT. 


\section{Percepção de estudantes ante processos de ensino- aprendizagem durante pandemia pela covid-19}

\section{Resumo}

A chegada da pandemia ocasionada pela covid-19 revolucionou o ensino universitário e modificou as estratégias pedagógicas utilizadas pelos professores bem como as de aprender dos estudantes, obrigando a uma transformação de uma modalidade presencial a uma intermediada por ambientes digitais. O objetivo deste estudo é descrever a percepção de estudantes da faculdade de saúde ante os processos de ensino-aprendizagem em aula invertida e aula magistral durante a pandemia pela covid-19. Num estudo misto de tipo descritivo de corte transversal, constituido por uma amostra não probabilística por conveniência de 74 estudantes, o instrumento utilizado foi o questionário estruturado, e a análise dos dados foi realizada pela estatística básica descritiva e pela análise do discurso. Os resultados evidenciaram que, nas aulas com formato de aula invertida, é promovida mais auto-organização, produto das aulas assincrônicas, além disso são gerados espaços de reflexão com relação às aprendizagens adquiridas por meio de fóruns e participações espontâneas na aula. Quanto às avaliações formativas e inclusive às sumativas, esse cenário é percebido pelos estudantes de forma positiva, e estes consideram que, a partir das aulas magistrais são cumpridos os objetivos de aprendizagem estipulados no currículo e uma aprendizagem significativa ocorre. Em conclusão, para os estudantes, a modalidade remota é uma estratégia útil que dá continuidade ao ensino universitário em tempos de pandemia, embora considerem que ela diminui o rigor do processo educacional.

\section{Palavbras-chave (Fonte: tesauro da Unesco) Aprendizagem; aula magistral; ensino; pandemia; TIC.}


Uno de los grandes retos que ha enfrentado la educación superior en Colombia es la deserción estudiantil. Así, para el periodo 2017-2 la tasa alcanzó el 42,36\%, según datos reportados por el Sistema para la Prevención y Análisis de la Deserción en las Instituciones de Educación Superior (Spadies) (Barbosa y Pinzón, 2018). El Ministerio de Educación Nacional (MEN) en 2010 consideró que la clave para asegurar la permanencia de los estudiantes está en poder detectar de manera temprana los casos más vulnerables y generar estrategias de atención para ellos, siendo la motivación un factor importante para finalizar con éxito sus programas académicos (Pascuas, Jaramillo y Verástegui, 2015). Por otro lado, Altbach et al. (2014) indicaron los retos que debía asumir el país a nivel educativo durante el periodo (2014-2024), condicionado por la globalización y las expectativas sociales, y aunque nunca previó una pandemia, estos retos eran en innovación, cobertura, financiación, calidad e internacionalización. Algunas de las estrategias institucionales implementadas para contrarrestar este fenómeno fueron la flexibilización de los programas académicos, con el fin de adaptarlos a las necesidades de tiempo y recurso de los estudiantes; la implementación de las tecnologías de la información y la comunicación (TIC), como complemento educativo para el desarrollo de sus cursos; y el desarrollo de otras metodologías, como la clase invertida, para garantizar el aprendizaje autónomo y significativo de los estudiantes (Turney, 2007).

Las TIC siempre han sido un reto en el proceso educativo. Desde 1998 la Unesco identificó su inclusión en la educación como una acción prioritaria (Vidal et al., 2004); sin embargo, en la actualidad no todas las instituciones de educación superior han logrado incorporar estas estrategias a sus programas académicos de manera contundente, ya sea por contar con recursos económicos escasos, por falta de entrenamiento o desconocimiento de herramientas digitales o por la preferencia docente por metodologías tradicionales, razones por las cuales la edu- cación apoyada en medios digitales aún presenta grandes dificultades en nuestro país.

Con la llegada de la pandemia por el virus SarsCoV-2, además de las medidas de confinamiento decretadas a nivel nacional, la educación universitaria se vio desafiada a mudar sus cursos de una modalidad presencial a una mediada por recursos digitales (Roa, Cobo y Gómez, 2020), medidas que revolucionaron la cotidianidad académica, ya que no todas las instituciones estaban preparadas para enfrentar un entorno educativo 100\% virtual o digital. Sin embargo, las instituciones universitarias han realizado grandes esfuerzos por plantear estrategias para continuar con el desarrollo y culminación de sus programas académicos durante esta pandemia.

En este contexto, la Pontificia Universidad Javeriana Cali centra su modelo educativo en el Paradigma Pedagógico Ignaciano (PPI) (Díaz, 2016), el cual refuerza la formación integral de los estudiantes con capacidad crítica para enfrentar las realidades sociales y los nuevos retos que implican transformaciones pedagógicas y adaptaciones al medio, incluso respecto de situaciones asociadas a una pandemia. En este sentido, el PPI se transforma y se articula con las realidades actuales, lo que incluye la labor de los profesores, quienes tuvieron que reinventar sus procesos didácticos y pedagógicos para afrontar las clases, además de realizar el acompañamiento a los estudiantes de forma remota (Moreno, 2020). Estas nuevas adaptaciones y cambios afectaron toda la dinámica universitaria, donde estudiantes y docentes debieron modificar sus rutinas, estrategias de enseñanza y aprendizaje con el fin de optimizar los nuevos procesos académicos implementados en la virtualidad (Rose, 2020).

En la Pontificia Universidad Javeriana Cali existen diferentes metodologías para los procesos de enseñanza-aprendizaje a través de medios remotos, entre las que se destacan la clase magistral y la clase invertida o flipped. La clase magistral se basa en el diseño e implementación de actividades donde el 
docente realiza una conferencia o disertación, generalmente apoyada en la proyección imágenes, y de esta manera se permite el contacto humano en las aulas de clase, manteniendo una mayor motivación de los estudiantes y una retroalimentación inmediata de los contenidos (Quijano, 2010). Sin embargo, al ser uno de los métodos más pasivos para el estudiante, su uso en la actualidad es controvertido, ya que se percibe como una acumulación de conocimiento donde el estudiante no tiene la oportunidad de explorar habilidades creativas que le permitan adaptarse a situaciones flexibles ni fortalecer el trabajo autónomo y colaborativo. Por su parte, el modelo de enseñanza-aprendizaje de clase invertida busca principalmente modificar los ambientes en los que se llevaban tradicionalmente a cabo los procesos académicos haciendo uso de las TIC y liberando el tiempo en el aula para promover un mayor acompañamiento del docente en actividades que permitan suplir las necesidades particulares y censar los avances de los estudiantes, lo cual potencia una participación más activa del estudiante tanto dentro como fuera del salón de clase, al verse expuesto a actividades que promueven un aprendizaje activo, significativo y colaborativo (Bergmann y Sams, 2014).

La transición desde la modalidad presencial hacia una remota puede estar condicionada por la capacidad tecnológica y las habilidades en el uso de herramientas y aplicaciones a través de medios digitales. Por esta razón, el objetivo de este estudio fue describir la percepción de los procesos de enseñanza-aprendizaje de los estudiantes de la Facultad de Salud de la Pontificia Universidad Javeriana Cali respecto de las metodologías de aula invertida versus la clase magistral en las circunstancias asociadas a la pandemia por el covid-19.

\section{Materiales y métodos}

El estudio, de tipo descriptivo y corte transversal, siguió la lógica del enfoque mixto de investigación, asumiendo posturas cuantitativas y cualitativas respecto de las realidades educativas de estudiantes de la Facultad de Salud de la Pontificia Universidad Javeriana Cali que devienen con la pandemia desatada por el virus Sars-CoV-2.

La población de estudio estuvo conformada por estudiantes matriculados en las carreras de Medicina, Enfermería, y Nutrición y Dietética durante el periodo 2020-1. Se realizó un muestreo no probabilístico por conveniencia. La muestra estuvo conformada por 74 estudiantes que cumplían con los criterios de inclusión: estar matriculados en una de las tres carreras de pregrado de la Facultad de Salud, aceptar participar voluntariamente en el estudio y responder el instrumento en su totalidad. Se excluyeron los datos de los participantes que no diligenciaron el cuestionario completamente. El instrumento utilizado fue un cuestionario estructurado con 41 preguntas en total: 40 cerradas y una abierta, la cual se enfocó en identificar y comprender las enseñanzas personales que dejó la pandemia por covid-19 en los estudiantes. Las preguntas cerradas se dividieron en tres apartados: el primer apartado recogió información sobre los datos sociodemográficos, uso de tecnologías de la información (computador, conexión a internet), habilidades y percepciones generales de los ambientes virtuales (15 preguntas); el segundo apartado recogió información sobre los procesos de enseñanza y aprendizaje en asignaturas con la metodología de aula invertida (13 preguntas); y el tercer apartado presentó preguntas sobre los procesos de enseñanza y aprendizaje, pero en las asignaturas con metodología de clase magistral (13 preguntas). La aplicación del instrumento se realizó empleando la plataforma Forms de Google.

A los estudiantes se les indicó que evaluaran simultáneamente la percepción del aprendizaje en ambas estrategias educativas. La estrategia de clase magistral debían evaluarla en un curso cuya metodología fuera de tipo "tradicional", mientras que la estrategia de clase invertida debía evaluarse con base en la experiencia propia durante los cursos con esta modalidad en el semestre 2020-1. 
Las preguntas que evalúan la percepción de los procesos de enseñanza y aprendizaje fueron procesadas, de acuerdo con la escala de Likert, de o a 4 (o: No Sabe/No Responde/No aplica, 1: Totalmente en desacuerdo, 2: En desacuerdo, 3: De acuerdo y 4:Totalmente de acuerdo). Para la validación del instrumento se calculó el coeficiente de alfa de Cronbach, que arrojó un valor de o,82, el cual es aceptable y permite asegurar la consistencia interna del instrumento. Los datos fueron tabulados y analizados a través de estadística descriptiva básica, y se determinó la media y la desviación estándar. Para los datos sociodemográficos, uso de tecnologías de la información, habilidades y percepciones generales de los ambientes digitales se determinaron frecuencias y porcentajes, expresados en tablas y gráficas. Para el análisis de percepción del aprendizaje y la enseñanza se calcularon los valores promedios de cada una de las 26 respuestas, los cuales se califican como "Positivo", si el promedio es $\geq 3$; "Puede mejorarse", si el promedio se encuentra entre 2 y $3, y$ "Negativo", si el promedio es $\leq 2$. La existencia de significatividad estadística en las diferencias entre las dos estrategias pedagógicas se analizó mediante la prueba $T$ de Students, asumiendo la normalidad de los datos. Un valor de $p<0,05$ se consideró estadísticamente significativo y los datos fueron analizados mediante el programa SPSS versión 23.

Para la pregunta abierta se logró identificar seis categorías de análisis relacionadas con el estudio. La información se procesó con el software de investigación cualitativa Atlas.TI, siguiendo la lógica de categorización abierta, axial y selectiva, la cual permitió develar las categorías finales con relación a las enseñanzas personales significativas. Cada categoría estuvo constituida por los diferentes relatos de los estudiantes, analizados en la lógica de análisis del discurso (Wodak y Meyer, 2003).

\section{Resultados}

En la Figura 1 se identifica el porcentaje de estudiantes por programa académico (Medicina, En- fermería, y Nutrición y Dietética) que participaron en el estudio. El promedio de edad fue de 18,4 años \pm 2,1 , donde el $81,1 \%$ corresponde al sexo femenino y el $18,9 \%$ al masculino.

Del total de la muestra, el o,9\% de los estudiantes mencionaron no tener computador en casa, el 33,4\% comparten equipo con alguna persona, mientras que el $65,6 \%$ indicaron contar con total disponibilidad de tiempo para su uso. Con relación a la calidad del internet, el 50\% de los estudiantes manifestaron tener una conexión a internet entre buena y excelente, mientras que la otra mitad presentan dificultades constantes con la conexión, tal como se presenta en la Figura 2.

El $85 \%$ de los estudiantes manifestaron estar al frente de la pantalla de su computador más de 6 horas al día, mientras que el 1,4\% afirmó estar frente al computador entre 1 y 3 horas al día, tal como se evidencia en la Figura 3.

\section{Uso de recursos tecnológicos}

Sobre el uso de herramientas digitales, el 56,8\% de los estudiantes consideró tener habilidades intermedias y $23 \%$ en habilidad básica. Solo el $20 \%$ de los estudiantes percibieron sus habilidades en un nivel avanzado. Sobre el nivel de habilidad en el aula virtual (Blackboard), el 52,7\% se ubicó en nivel intermedio y el 20,3\% en nivel básico.

Con la llegada de la educación remota, el 48,7\% de los estudiantes manifestaron no estar preparados para la nueva realidad, mientras que el 51,3\% manifestó estar en condiciones de asumir la educación a través de medios digitales (Figura 4).

En la Figura 5 se observa que el tiempo de dedicación al proceso de formación en el semestre fue definido por la mayoría de los estudiantes entre 9 y 12 horas diarias $(44,6 \%)$ y entre 6 y 9 horas con un $31,1 \%$ de la población. El $50 \%$ de los estudiantes considera que dedica mayor tiempo al estudio de manera presencial, mientras para la otra mitad su mayor dedicación fue en la modalidad remota. 
ISSN 0123-1294 | e-ISSN 2027-5358 | Educ.Educ. Vol. 24. No. 2 | Mayo-julio de 2021 | pp. 199-217

Universidad de La Sabana | Facultad de Educación

Figura 1. Distribución de los participantes por programa académico

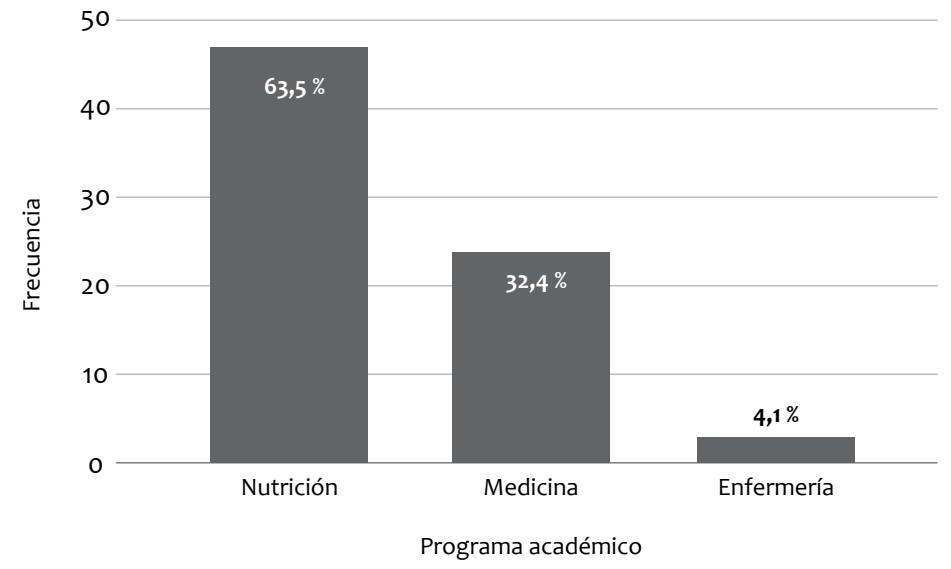

Fuente: elaboración propia.

Figura 2. Calidad de conexión a internet

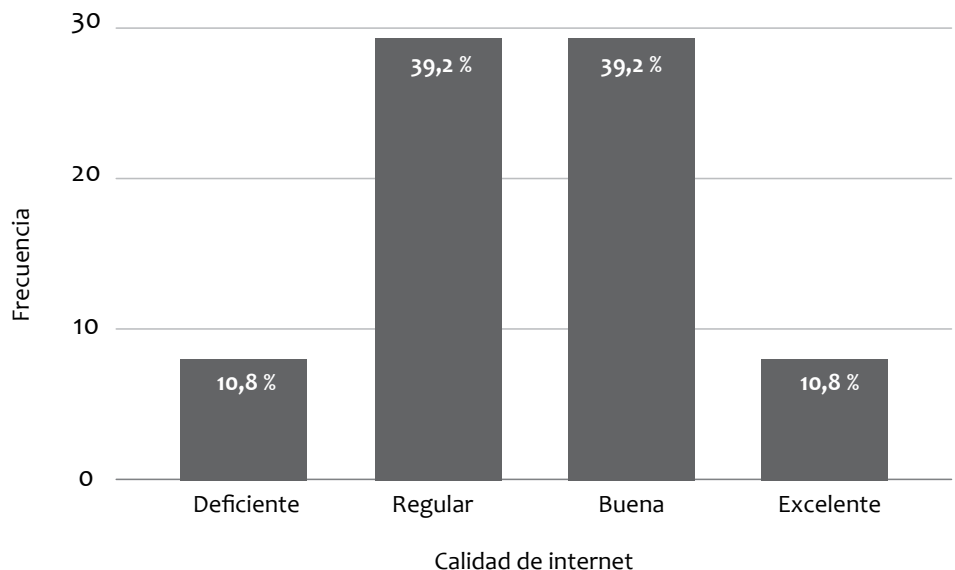

Fuente: elaboración propia.

Figura 3. Rango horario de uso del computador

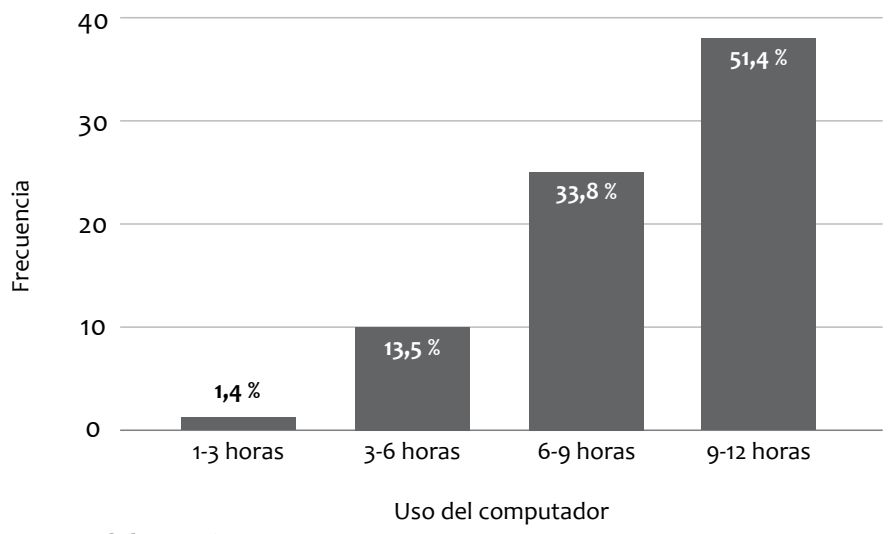

Fuente: elaboración propia. 
Tabla 1. Comparativo de habilidades entre herramientas digitales y Blackboard

\begin{tabular}{|c|c|c|c|c|}
\hline \multirow{2}{*}{} & \multicolumn{2}{|c|}{ Herramientas digitales } & \multicolumn{2}{c|}{ Blackboard } \\
\cline { 2 - 5 } & Frecuencia & Porcentaje & Frecuencia & Porcentaje \\
\hline Básicas & 17 & $23,0 \%$ & 15 & $20,3 \%$ \\
\hline Intermedias & 42 & $56,8 \%$ & 39 & $52,7 \%$ \\
\hline Avanzadas & 15 & $20,3 \%$ & 20 & $27 \%$ \\
\hline Total & 74 & $100 \%$ & 74 & $100 \%$ \\
\hline
\end{tabular}

Fuente: elaboración propia.

Figura 4. Preparación frente a la metodología remota

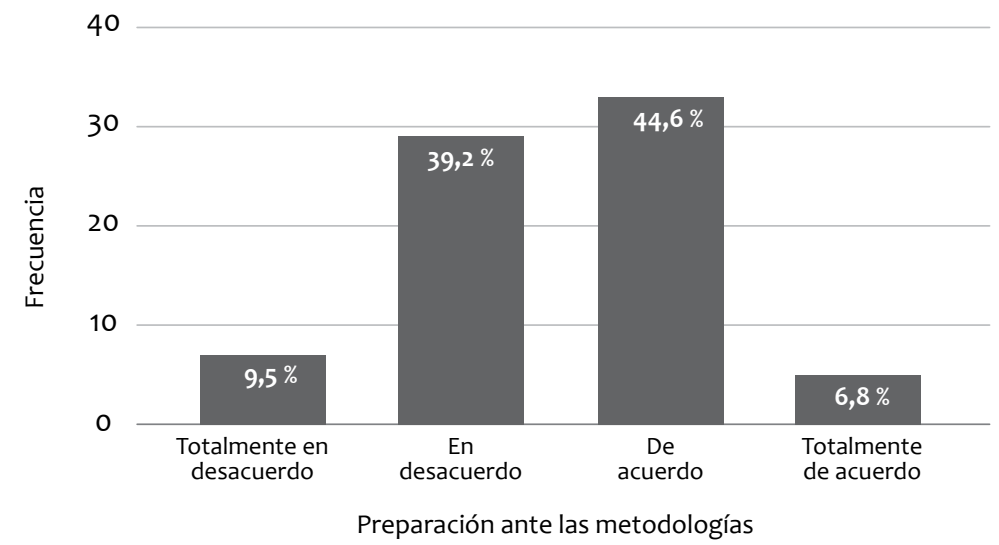

Fuente: elaboración propia.

Figura 5. Tiempo de dedicación al proceso de formación en el semestre

$$
40
$$$$
30
$$

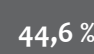

$$
\text { 즘 }
$$

20

$31,1 \%$

选

10

$21,6 \%$

$2,7 \%$

0

1-3 horas 3-6 horas

6-9 horas

9-12 horas

Tiempo de dedicación

Fuente: elaboración propia. 
El 68,9\% de los estudiantes consideraron parcial o totalmente que la educación de manera remota disminuyó el rigor académico, frente al 31,1\% que no consideran que su rigor académico se haya visto afectado (Figura 6).

En la Tabla 2 se aprecian las diferentes percepciones de los estudiantes frente a la clase magistral y la clase invertida en los aspectos que involucran el aprendizaje y la enseñanza. Con respecto a las preguntas de la encuesta correspondientes al apartado de aprendizaje, se evidenció que los objetivos se cumplieron con ambos modelos, siendo el modelo de clase magistral el que registra valores promedio más favorables $(p=0,029)$. Por otra parte, los estudiantes consideran en mayor medida que los encuentros tanto sincrónicos como asincrónicos con sus docentes son necesarios para alcanzar los objetivos de aprendizaje en la modalidad de clase invertida $(p \leq 0,001)$. El aprendizaje significativo, autónomo y colaborativo no presenta diferencias estadísticamente significativas entre las metodologías analiza$\operatorname{das}(p>0,1)$.

Figura 6. Rigor académico

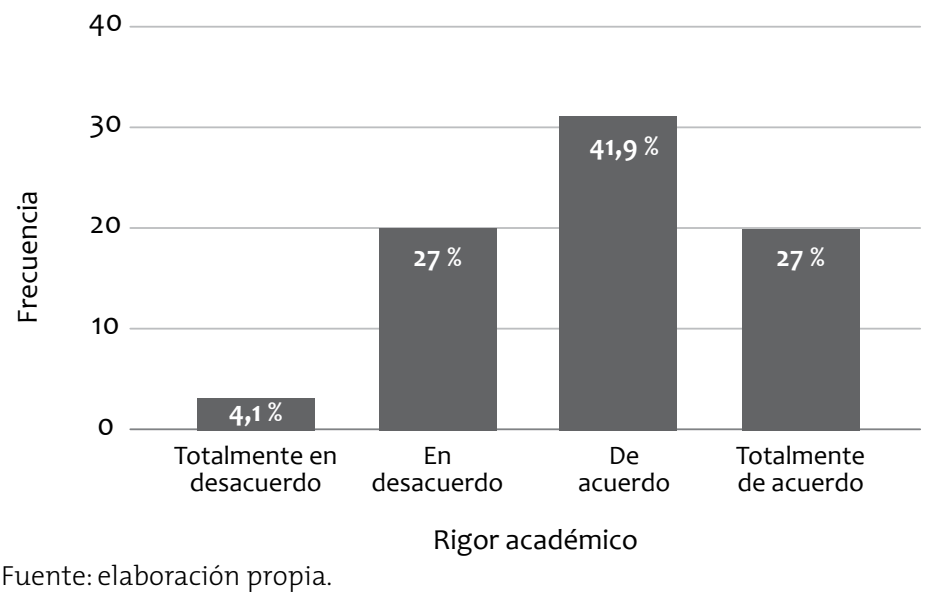

Tabla 2. Puntuaciones medias y desviaciones típicas (DT) por pregunta sobre la percepción de la clase magistral y de clase invertida

\begin{tabular}{|c|c|c|c|c|c|c|c|}
\hline & & \multicolumn{2}{|c|}{ Clase magistral } & \multicolumn{2}{|c|}{ Clase invertida } & \multirow{2}{*}{$\mathbf{t}$} & \multirow{2}{*}{$p$} \\
\hline & & Media & DT & Media & DT & & \\
\hline \multirow{6}{*}{$\begin{array}{l}\frac{U}{N} \\
\text { N } \\
\frac{0}{0} \\
\frac{0}{0} \\
\frac{0}{2}\end{array}$} & $\begin{array}{l}\text { ¿Usted considera que se cumplieron los objetivos de aprendizaje estipulados en el } \\
\text { syllabus durante la pandemia de covid-19? }\end{array}$ & 3,43 & 0,64 & 3,19 & 0,61 & $-2,357$ & $0,029^{*}$ \\
\hline & $\begin{array}{l}\text { ¿Considera que los encuentros sincrónicos (usando plataformas digitales como } \\
\text { Collaborate, Zoom, Google Meet) con el docente fueron un espacio indispensable para } \\
\text { alcanzar sus objetivos de aprendizaje durante la pandemia de covid-19? }\end{array}$ & 3,01 & 1,26 & 3,53 & 0,58 & 3,196 & ০,০००* \\
\hline & $\begin{array}{l}\text { ¿Considera que los encuentros asincrónicos (usando plataformas digitales como foros, } \\
\text { wikis, clases grabadas) con el docente fueron indispensables para alcanzar sus objetivos } \\
\text { de aprendizaje durante la pandemia de covid-19? }\end{array}$ & 2,89 & 1,33 & 3,19 & 0,77 & 1,664 & $0,001^{*}$ \\
\hline & $\begin{array}{l}\text { ¿Considera que su aprendizaje ha sido significativo (capacidad de asociar la información } \\
\text { nueva con la que posee, guiado por el docente) durante la pandemia de covid-19? }\end{array}$ & 3,18 & 0,67 & 3,01 & 0,67 & $-1,470$ & 0,156 \\
\hline & $\begin{array}{l}\text { ¿Considera que la manera como se desarrolla el curso incentiva su aprendizaje } \\
\text { autónomo (capacidad de construir conocimiento por sí mismo, guiado por el docente) } \\
\text { durante la pandemia de covid-19? }\end{array}$ & 3,12 & 0,76 & 2,97 & 0,79 & $-1,166$ & 0,460 \\
\hline & $\begin{array}{l}\text { ¿Considera que las actividades grupales de aprendizaje colaborativo permitieron } \\
\text { mejorar su comprensión a la luz de los objetivos de aprendizaje durante la pandemia de } \\
\text { covid-19? }\end{array}$ & 3,11 & 0,69 & 3,11 & 0,59 & 0,000 & 0,460 \\
\hline
\end{tabular}

* Significancia estadística obtenida mediante la prueba $t$ de Student.

Fuente: elaboración propia. 
En cuanto a la enseñanza, los resultados de la percepción de los estudiantes acerca de los recursos educativos y estrategias de evaluación sumativa y formativa ofrecidos son estadísticamente diferentes $(p \leq 0,014)$ en ambas modalidades de clase. Se obser- vó que los valores de desviación típica de los datos presentan una tendencia más alta en algunos aspectos evaluados en la modalidad de clase magistral. Esto indica una mayor dispersión en las respuestas de los estudiantes en esta modalidad.

Tabla 3. Puntuaciones medias y desviaciones típicas (DT) por pregunta sobre la percepción de la clase magistral y de clase invertida

\begin{tabular}{|c|c|c|c|c|c|c|c|}
\hline & \multicolumn{2}{|c|}{ Clase magistral } & \multicolumn{2}{|c|}{ Clase invertida } & \multirow{2}{*}{$\mathbf{t}$} & \multirow{2}{*}{$p$} \\
\hline & & Media & DT & Media & DT & & \\
\hline \multirow{7}{*}{ 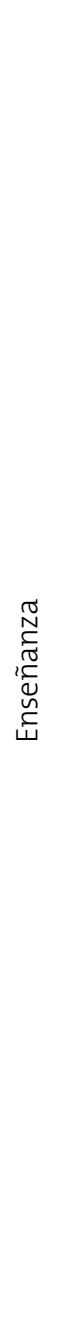 } & $\begin{array}{l}\text { ¿Considera que el tiempo de acompañamiento } \\
\text { docente (antes, durante y después) fue suficiente } \\
\text { para sentirse apoyado durante el proceso enseñanza- } \\
\text { aprendizaje durante la pandemia de covid-19? }\end{array}$ & 3,19 & 0,75 & 3,41 & 0,59 & 1,939 & 0,236 \\
\hline & $\begin{array}{l}\text { ¿Considera que las actividades de discusión, debate } \\
\text { y /o retroalimentación lo llevaron a desarrollar } \\
\text { procesos reflexivos frente a las temáticas trabajadas } \\
\text { en el curso durante la pandemia covid-19? }\end{array}$ & 3,18 & 0,65 & 3,22 & 0,67 & 0,375 & 0,953 \\
\hline & $\begin{array}{l}\text { ¿Considera que las estrategias de evaluación } \\
\text { sumativa (exámenes, parciales y finales) dieron } \\
\text { cuenta del alcance de los objetivos de aprendizaje } \\
\text { durante la pandemia de covid-19? }\end{array}$ & 3,15 & 1,03 & 3,23 & 0,59 & 0,589 & $0,014^{*}$ \\
\hline & $\begin{array}{l}\text { ¿Considera que las estrategias de evaluación } \\
\text { formativa (clubes de revistas, modelos, retos lúdicos, } \\
\text { seminarios, foros, casos, construcción de modelos) } \\
\text { dieron cuenta del alcance de los objetivos de } \\
\text { aprendizaje durante la pandemia covid-19? }\end{array}$ & 2,65 & 1,43 & 3,15 & 0,57 & 2,800 & ০,०००* \\
\hline & $\begin{array}{l}\text { ¿Considera que las estrategias de evaluación } \\
\text { diagnóstica (actividades de comprobación de } \\
\text { lectura) dieron cuenta del alcance de los objetivos de } \\
\text { aprendizaje durante la pandemia de covid-19? }\end{array}$ & 2,99 & 0,77 & 3,19 & 0,63 & 1,752 & 0,783 \\
\hline & $\begin{array}{l}\text { ¿Considera que los recursos educativos previos } \\
\text { a las clases (lecturas, talleres, mapas, videos) } \\
\text { fueron suficientes y pertinentes para favorecer su } \\
\text { rendimiento académico durante la pandemia de } \\
\text { covid-19? }\end{array}$ & 2,41 & 1,45 & 3,15 & 0,79 & 3,870 & $0,000^{*}$ \\
\hline & $\begin{array}{l}\text { ¿Considera que el contenido temático y los } \\
\text { conocimientos adquiridos tienen aplicación en un } \\
\text { contexto real, dado su perfil profesional, durante la } \\
\text { pandemia de covid-19? }\end{array}$ & 3,45 & 0,62 & 3,32 & 0,62 & $-1,189$ & 0,893 \\
\hline
\end{tabular}

* Significancia estadística obtenida mediante la prueba t de Student.

Fuente: elaboración propia. 
Para comprender las enseñanzas personales significativas que dejó la pandemia por covid-19 en los estudiantes de las carreras de Salud, se logró identificar seis categorías de análisis: disciplina y autonomía, adaptación al cambio, responsabilidad, organización del tiempo, habilidades en el uso de herramientas digitales y solidaridad, tal como se presentan en la Figura 7.

Las categorías que emergen con mayor fuerza social son la disciplina y la autonomía. Se lograron identificar 38 relatos relacionados con las enseñanzas significativas que les dejó la pandemia a los estudiantes de las carreras de salud; entre ellos, se encontraron: "Académicamente ha sido un reto estudiar desde la casa, pues se presentan muchas distracciones, pero la enseñanza más significativa es que pude coger disciplina de levantarme a clase de 7 am y seguir estudiando derecho como si estuviera en la U"

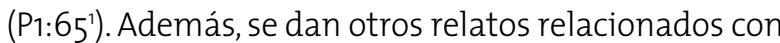
esta categoría: "El autodisciplinarse y tener compromiso conmigo misma fue una excelente estrategia para obtener excelentes frutos" (P1:30). Es así como se logró evidenciar la disciplina y autonomía en los estudiantes.

La categoría de adaptación al cambio fue otra de las que emergió con gran fuerza social (24 relatos) entre los estudiantes, así: "El desarrollo de autonomía para el logro de los objetivos académicos, estando en casa, se torna más complejo por las distracciones del hogar, o conversaciones con la familia; de cierto modo, a mi parecer, el hogar es para descansar y dispersarse, pero esto se debe modificar para cumplir con las metas" (P1:35).

La categoría responsabilidad sobresale como uno de los aprendizajes personales significativos entre los estudiantes en este tiempo de pandemia, donde se lograron evidenciar relatos como: "La autonomía y responsabilidad es la clave del aprendizaje, más en tiempos de virtualidad" (P1:10); "Nos enseña a exigirnos un poquito más a pesar de los distractores que haya y que no es fácil, pero toca mirar alternativas para un método de estudio" (P1:36).

Para algunos estudiantes, el aprendizaje significativo se observó en la interacción y uso de nuevas herramientas digitales, evidenciados en los siguientes relatos: "La enseñanza académica más significativa que ha dejado esta pandemia del covid-19 fue el aprendizaje en cuanto al manejo de herramientas virtuales que antes no conocía y no tenía la capacidad de usar" (P1:40); "Aprender nuevos mecanismos de aprendizaje virtuales" (P1:1). Donde la inclusión del aprendizaje a través del aula invertida puede generar nuevas formas de adquirir conocimiento.

Figura 7. Enseñanzas personales significativas que deja la pandemia por covid-19

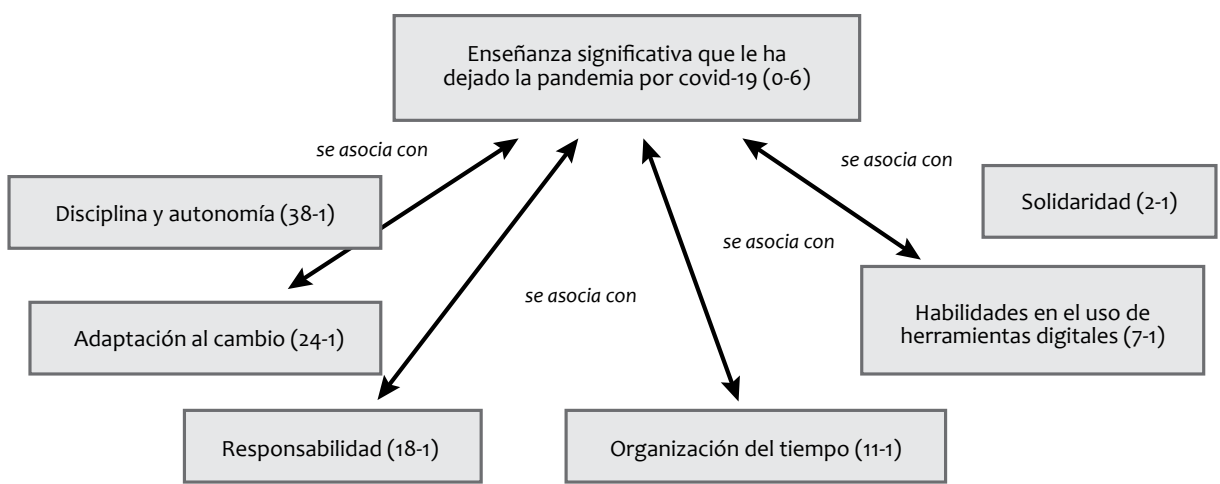

Fuente: elaboración propia

$\mathrm{P} 1: 65 \mathrm{P}=$ Primary document (documento primario $=1$ ), $65=$ Número del relato. 


\section{Discusión}

Alcanzar la comunicación a través de internet requiere de infraestructura física y tecnológica, así como equipos informáticos (Villatoro y Silva, 2005). En este estudio se encontró que el 50\% de los estudiantes presentan problemas de conexión a internet, calificándola como regulary deficiente, e incluso no cuentan con ella. Estos datos son significativos, e indican que la información no llega a todas las personas, lo cual limita su participación en el proceso de intercambio, debate y crecimiento colectivo, en directa contravía con el derecho humano a la información (Casillas, 2019), más aún cuando la educación, que es otro derecho fundamental, ha migrado a modalidad virtual o remota asociada al aislamiento social y al confinamiento obligatorio producto de la pandemia por covid-19. La dificultad y/o ausencia de acceso a los recursos tecnológicos o de conexión a internet generan desigualdad digital (DiMaggio y Hargittai, 2001), pues la cobertura es insuficiente e inestable en diferentes sectores del país, de modo que aumenta la violencia estructural (Tinoco, Osorio y González, 2019). Por todo ello, es indispensable continuar desarrollando acciones encaminadas a subsanar estas desigualdades digitales.

En este estudio se evidencia que el 50\% de los estudiantes tienen computador propio y no tienen que compartirlo con nadie, mientras que el 34,3\% deben compartirlo con otros miembros de la familia, situación que los llevó a desarrollar estrategias para el uso de estos dispositivos que conllevan limitaciones en su uso en actividades académicas y laborales por los miembros del hogar. Tales datos se encuentran por debajo de los reportados por De León, Tapia y Vélez (2020), en cuyo estudio 73\% de los estudiantes comparen el computador entre uno o más miembros de la familia. Un sondeo realizado en una universidad pública de México evidencia que, en promedio, $73,6 \%$ de los estudiantes universitarios se conectan y usan sus computadores diariamente durante 4 horas o más (Morales et al., 2020). De León et al. (2020) mencionan que $68 \%$ de los estudiantes utilizan el computador más de 6 horas. Comparado con los resultados de este estudio, en el que se observa que $85,2 \%$ usan el computador durante 6 horas o más, estos datos difieren del estudio de Rodrigues et al. (2009), quienes reportaron que los estudiantes solo se conectan una vez a la semana y desde computadores ajenos, evidenciando un crecimiento del uso de internet y de aparatos electrónicos propios, como el computador, durante la última década.

La implementación de las TIC dentro de los procesos de enseñanza-aprendizaje permite desarrollar diferentes estrategias exitosas para el desarrollo de contenidos y el favorecimiento del trabajo de los estudiantes, al optimizar el tiempo de dedicación (Cabero, Del Prete y Arancibia, 2019; Valencia, Valencia y Gómez, 2021), sobre todo en los estudiantes actuales, denominados "nativos digitales", que según Prensky (2001) son aquellas personas nacidas desde 1997 en adelante. Sin embargo, esto no garantiza que los estudiantes tengan habilidades en el uso de las TIC asociadas al desarrollo de situaciones complejas, con análisis e interpretación propios de un proceso académico, debido a que los estudiantes prestan menos atención a los contenidos, por la gran cantidad de datos a los que tienen acceso (Delgado, 2020). Esto respalda los resultados obtenidos con relación al uso de herramientas digitales, en donde solo 20,3\% mencionó tener habilidades avanzadas y $23 \%$ tienen habilidades básicas, mientras que para el uso de la plataforma institucional Blackboard 27\% considera tener habilidades avanzadas y 20,3\% habilidades básicas, a pesar de considerarse nativos digitales.

Dadas las características asociadas a la pandemia por covid-19, todas las instituciones de educación superior se vieron obligadas a acoger la modalidad remota como la nueva y única realidad para culminar sus cursos. Este repentino cambio implicó una serie de estrategias para acoplarse al escenario digital y ofrecer a los estudiantes la misma exigencia y calidad académica. Frente a esta situación, Rose (2020) concluyó que toda la comunidad académica tuvo y tendrá que seguir aprendiendo de la expe- 
riencia, priorizando estrategias innovadoras para cumplir con los objetivos de aprendizaje, al ritmo de los picos epidemiológicos del virus. El 51,4\% de los estudiantes consideró que se sintieron preparados parcial o totalmente para el cambio de modalidad educativa, y esta percepción puede estar muy ligada a las necesidades propias de aprendizaje, la red de apoyo previamente establecida entre estudiantes y profesores, las habilidades y experiencia en medios digitales y las condiciones técnicas y económicas de cada persona (Jennifer, 2007). Estas mismas situaciones pueden influir en la dedicación horaria. En este estudio se evidenció que $75,7 \%$ de los estudiantes indicó rangos entre las 6 y 12 horas diarias, y se debe considerar que las actividades propias del hogar pueden generar interrupciones repetitivas y, con ello, mayor dificultad para concentrarse en el estudio.

A pesar de que $77,1 \%$ de los estudiantes contaban con habilidades entre intermedias y avanzadas en recursos tecnológicos y 75,7\% dedicó entre 6 y 12 horas diarias a su proceso de formación, 68,9\% consideró que el rigor académico disminuyó ante la transición de modalidad presencial a digital asociada a la pandemia. Esta información indica que la mayoría de los sistemas de educación no están siendo efectivos, ya que replican la forma como se ha aprendido históricamente, trasladando la problemática de la educación tradicional. La educación a distancia debe verse como un acuerdo didáctico y dinámico entre el profesor y el estudiante, donde este último aprende de forma autónoma y colaborativa, aunque se encuentren en espacios diversos. De esta manera, la interacción, como función central en los ambientes de aprendizaje, requiere al menos dos objetos y dos acciones que participen recíprocamente. Estas interacciones, reconocidas como componentes de la educación a distancia, son: estudiante-estudiante, estudiante-profesor y estudiante-contenido. Según Anderson (2003), el aprendizaje profundo y significativo se logra cuando alguna de las tres interacciones se encuentra en niveles superiores y cuando convergen varias de ellas. Estas in- teracciones se ven altamente representadas en los modelos de aprendizaje de clase invertida (Gilboy, Heinerichs y Pazzaglia, 2015).

Así mismo, Moreira et al. (2014) establecen que la labor docente debe reconocer esa necesidad de mediación y facilitar la construcción diaria de experiencias en el aula física o virtual de clase. De allí que sea fundamental la planificación del aprendizaje por medio electrónico y las estrategias de apoyo que permitan conformar escenarios de interacción y mantener la exigencia académica (Link y Marz, 2006). Otros factores a tener en cuenta son las preferencias de aprendizaje de los estudiantes y su actitud frente al proceso, ya que algunos estudios reportan que los estudiantes independientes y autónomos tienen mayor aceptación de la modalidad digital, lo que implica el éxito de la misma (Horiuchi et al., 2009) y se relaciona con lo descrito por Juca (2016), quien resalta como elemento fundamental el conocimiento del estudiante acerca de sus saberes reales, potencialidades y limitaciones, para poder trazar su propia ruta de aprendizaje, aspectos típicos de los modelos de aprendizaje invertido.

Con relación al uso de recursos educativos previos a las clases (lecturas, talleres, mapas y videos) y de estrategias de evaluación formativa y sumativa, los resultados de este estudio evidenciaron que se favoreció el aprendizaje autónomo y significativo en la metodología de clase invertida durante tiempos de pandemia (Bishop y Verleger, 2013). Tune, Sturek y Basile (2013) sustentan que el uso del modelo de clase invertida permite mayores aprendizajes con respecto a la clase magistral. Es de destacar que su aplicación requiere de una planeación rigurosa y estructurada del curso donde las herramientas pedagógicas y didácticas deben estar alineadas con los objetivos de aprendizaje, cuidando que los recursos educativos sean suficientes y pertinentes, para evitar abrumar al estudiante (Mason, Shuman y Cook, 2013). Alcanzar todos los componentes de esta metodología implica que el docente se actualice en el desarrollo e implementación de la misma (Moreno, 2020). 
A pesar de que en el modelo de clase invertida el docente no es el protagonista, su acompañamiento continuo en cada uno de los momentos de la clase cobra vital importancia-como lo plantean Galindo y Quintana (2016) -y, además, ofrece una experiencia más interactiva entre docente y estudiante (Galway et al., 2014). Es por eso que los resultados de este estudio no muestran diferencias significativas entre ambos modelos con respecto al acompañamiento brindado por el docente. Claro está que la motivación y el interés del estudiante juegan un papel indispensable en el aprendizaje y la apropiación de un modelo pedagógico, sea invertido o magistral, y en un modelo de clase invertida exitoso se requiere que el estudiante se involucre por completo en su proceso de aprendizaje, situación que no es fácil de conseguir. De ahí que se pueda afirmar que la utilidad de este modelo es directamente proporcional a la implicación e intereses de los estudiantes (Kakosimos, 2015).

Los estudiantes refieren que en ambos modelos se cumplieron los objetivos de aprendizaje; sin embargo, hacen referencia a una disminución del rigor académico en el periodo de transición hacia la digitalización asociada a la pandemia por covid-19. Se puede inferir que ese rigor está siendo percibido atendiendo a las características y condiciones de acceso a los recursos tecnológicos, más que a los procesos de enseñanza-aprendizaje empleados en ambos modelos pedagógicos. La forma como el estudiante universitario concibe el aprendizaje depende del uso de las TIC y de un proceso personal, más o menos complejo, donde el resultado puede ser un aprendizaje profundo o meramente superficial (Díaz et al., 2020).

Por otro lado, las enseñanzas significativas que se lograron captar en los estudiantes están asociadas a las características propias de las carreras de Salud, sustentadas en la disciplina, autonomía, responsabilidad, organización del tiempo, capacidad para adaptarse al cambio y la solidaridad, como pilares de su formación y preparación para una vida productiva y exigente, como lo es el área de la salud (Juca, 2016).
En este sentido, la disciplina no se asumió en este trabajo como un comportamiento regido por la vigilancia y el castigo (Foucault, 2002), sino por las normas instituidas entre los estudiantes y el profesor, y se leyó como la capacidad de los estudiantes para organizar su trabajo en términos de dedicación y esfuerzo, alejados de los diferentes métodos poco efectivos para llamar al orden, y como la actitud para cumplir con las obligaciones propias de la vida académica: organizar los tiempos para preparar exposiciones, realizar trabajos en horarios establecidos, participar en foros, presentar casos clínicos, estudiar para los parciales, entre otros, que son vitales en su proceso académico, tal como lo establece Stanislavski: "Si deseas alcanzar cualquier cosa que quieras en la vida, debes tener disciplina y orden. Y debes empezar con tu propia autodisciplina" (2016, p. 16). La autonomía permite la autoorganización, que es indispensable en los procesos educativos. En ese sentido, algunos autores afirman que la transformación vital de los aprendizajes se da a partir de la autopoiesis y la autoorganización, la cual favorece el aprendizaje teniendo como centro la vida misma (Maturana y Varela, 1994). Y fue así como la pandemia modificó los aprendizajes y contenidos, donde los estudiantes tuvieron una gran capacidad autopoietica para asumir las diversas adaptaciones. Igualmente, el aula invertida ha ido consolidando otras transformaciones en los procesos educativos, entre ellas, las digitales (Diez, 2020), con adaptaciones en las diferentes metodologías utilizadas por los docentes (Aristega et al., 2020).

Para los estudiantes, la transformación digital implicó retos en la adquisición de nuevas habilidades tecnológicas, hasta transformar los aprendizajes previos con la adquisición de los nuevos saberes, todo ello producto de la utilización de nuevas plataformas y formas de construir su aprendizaje (Rose, 2020). El aula invertida favoreció el autoaprendizaje a través de la red y permitió un aprendizaje continuo, inclusive en los tiempos en que el profesor no estaba presente. Las enseñanzas personales signifi- 
cativas se enfocaron en la posibilidad de transformar las realidades de los estudiantes que participaron en esta investigación y lograron aprendizajes referidos no solo a los diferentes contenidos curriculares, sino a las realidades que implica la educación remota producto de la pandemia por covid-19.

En conclusión, los estudiantes que participaron en este estudio reconocen el esfuerzo y el trabajo realizados por los profesores para dar continuidad a las actividades académicas ante la transición de la educación apoyada en medios digitales; así mismo, identificaron como enseñanzas personales significativas que la disciplina y la autonomía son fundamentales para el desarrollo de sus actividades académicas, más aún en tiempos de pandemia, con lo que se evidenció una mayor responsabilidad en la organización de las actividades académicas apoyadas en medios digitales, incluso cuando perciben que la situación actual generó una disminución en el rigor académico.
Para los estudiantes no existe tanta diferencia entre las metodologías aplicadas (clase invertida y magistral) en relación con el aprendizaje significativo, autónomo y colaborativo en ambientes de enseñanza remota durante la pandemia. Además, consideran que la forma de evaluación -sumativa o formativaaplicada en las diferentes metodologías permitió alcanzar los objetivos planteados según las intencionalidades formativas de cada curso analizado.

Se recomienda continuar realizando estudios investigativos donde se logre involucrar un mayor número de población, con el fin de evidenciar la autorregulación en el uso del tiempo, la planificación de las actividades académicas durante la educación remotay su impacto en los procesos de enseñanza-aprendizaje.

\section{Declaración de conflictos de intereses}

Los autores no presentan ningún conflicto de intereses.

\section{Referencias}

Altbach, P. et al. (2014). Educación superior en Colombia: Doce propuestas para la próxima década. Barranquilla: Universidad del Norte. http://www.jstor.org/stable/j.ctt1c3qo1m

Anderson, T. (2003). Modes of interaction in distance education: Recent developments and research questions. Hand-book of distance education (pp. 129-144). Disponible en Academia.com. https://www.academia. edu/704638/Modes_of_interaction_in_distance_education_Recent_developments_and_research_questions

Aristega, A. M. M., Aristega, J. E. M., Angulo, R. J. C. y Masacon, M. R. H. (2020). Enseñar y aprender en tiempos de covid-19. Pro Sciences: Revista de Producción, Ciencias e Investigación, 4(34), 79-86. Recuperado de: http:// www.journalprosciences.com/index.php/ps/article/view/281

Barbosa-Fontecha, I. L. y Pinzón Plazas, D. M. (2018). Incidencia de la financiación en la deserción Universitaria en Bogotá. [Trabajo de Grado] Programa de Economía, Facultad de Ciencias Económicas y Administrativas, Universidad Católica de Colombia, Bogotá.

Bergmann, J. y Sams, A. (2014). Flipped learning: Maximizing face time. T+D, 68(2), 28-31.

Bishop, J. L. y Verleger, M. A. (2013). The flipped classroom: A survey of the research. $120^{\text {th }}$ ASEE National Conference Proceedings, Atlanta, GA, Paper ID \#6219, 1-18. DOI: https://doi.org/10.18260/1-2--22585 
Cabero, J., Del Prete, A. y Arancibia, M. L. (2019). Percepciones de estudiantes universitarios chilenos sobre el uso de redes sociales y trabajo colaborativo. RIED. Revista Iboeroamericana de Educación a Distancia, 22(2), 3555. DOI: https://doi.org/10.5944/ried.22.2.22847

Casillas, M. G. A. (2019). Desigualdad en el acceso a internet en México y la afectación en el ejercicio del derecho humano a la información. Nuevo Derecho, 15(24), 55-70. DOI: https://doi.org/10.25057/2500672X.1235

De León-Vázquez, I. I., Tapia-Castillo, D. I. y Vélez-Díaz, D. (2020). Estudio sobre los retos de la educación presencial a nivel superior ante la contingencia sanitaria del covid-19. XIKUA. Boletín Científico de la Escuela Superior de Tlahuelilpan, 8(16), 5-8. DOI: https://doi.org/10.29057/xikua.v8i16.5862

Delgado, M. V. S. (2020). Convergencia entre Nativos Digitales e Inmigrantes Digitales. Sinergias Educativas, 5(1). DOI: https://doi.org/10.37954/se.v5i1.109

Díaz-García, l., Cerveró, G. A., Suárez-Rodríguez, J. y Alonso, N. O. (2020). La relación entre las competencias TIC, el uso de las TIC y los enfoques de aprendizaje en alumnado universitario de educación. Revista de Investigación Educativa, 38(2), 549-566. DOI: https://doi.org/10.6018/rie.409371

Díaz-Duran, M. E. (2016). ¿Es compatible la educación a distancia con la pedagogía ignaciana? Journal of Technology Management \& Innovation, 11(1), 36-47. DOI: https://doi.org/10.4067/S0718-27242016000100006

Diez-Canseco, R. (2020). Transformación digital en la educación en tiempos del covid-19. 1 ed. La Molina, Perú: Universidad San Ignacio de Loyola.

DiMaggio, P. y Hargittai, E. (2001). From the "digital divide" to "digital inequality": Studying Internet use as penetration increases. Working Paper \#15, Center for Arts and Cultural Policy Studies, Woodrow Wilson School, Princeton University. Recuperado de: https://culturalpolicy.princeton.edu/sites/culturalpolicy/files/wp15_dimaggio_hargittai.pdf

Foucault, M. (2002). Vigilar y castigar: nacimiento de la prisión. 1, ed. Buenos Aires: Siglo XXI.

Galindo, J. J. y Quintana, M. G. B. (2016). Innovación docente a través de la metodología flipped classroom: percepción de docentes y estudiantes de educación secundaria. Didasc@lia: Didáctica y Educación, 7(6), 153-172. Disponible en: https://dialnet.unirioja.es/servlet/articulo?codigo $=6672798$

Galway, L. P., Corbett, K. K., Takaro, T. K., Tairyan, K. y Frank, E. (2014). A novel integration of online and flipped classroom instructional models in public health higher education. BMC Medical Education, 14(1), 181. DOI: https://doi.org/10.1186/1472-6920-14-181

Gilboy, M. B., Heinerichs, S. y Pazzaglia, G. (2015). Enhancing student engagement using the flipped classroom. Journal of Nutrition Education and Behavior, 47(1), 109-114. https://doi.org/10.1016/j.jneb.2014.08.008

Horiuchi, S., Yaju, Y., Koyo, M., Sakyo, Y. y Nakayama, K. (2009). Evaluation of a web-based graduate continuing nursing education program in Japan: A randomized controlled trial. Nurse Education Today, 29(2), 140-149. DOI: https://doi.org/10.1016/j.nedt.2008.08.009 
Jennifer, L. (2007). Computer mediated conferencing-a hope or hype for healthcare education in higher learning? A review of the literature. Nurse Education Today, 27(4), 318-324. DOI: https://doi.org/10.1016/j. nedt.2006.05.012

Juca, F. X. (2016). La educación a distancia, una necesidad para la formación de los profesionales. Revista Universidad y Sociedad, 8(1), 106-111. Recuperado de: http://scielo.sld.cu/pdf/rus/v8n1/rus15116.pdf

Kakosimos, K. E. (2015). Example of a micro-adaptive instruction methodology for the improvement of flippedclassrooms and adaptive-learning based on advanced blended-learning tools. Education for Chemical Engineers, 12, 1-11. DOI: https://doi.org/10.1016/j.ece.2015.06.001

Link, T. M. y Marz, R. (2006). Computer literacy and attitudes towards e-learning among first year medical students. BMC Medical Education, 6(1), 34. DOI: https://doi.org/10.1186/1472-6920-6-34

Maturana Romesín, H. y Varela García, F. (1994). De máquinas y seres vivos: autopoiésis: la organización de lo vivo. 5 ed. Chile: Editorial Universitaria.

Mason, G. S., Shuman, T. R. y Cook, K. E. (2013). Comparing the effectiveness of an inverted classroom to a traditional classroom in an upper-division engineering course. IEEE Transactions on Education, 56(4), 430-435. DOI: https://doi.org/10.1109/TE.2013.2249066

Morales, A., Zacatenco, J. D., Luna, M., García, R. Z. e Hidalgo, C. (2020). Acceso y actitud del uso de Internet entre jóvenes de educación universitaria. Revista Digital de Investigación en Docencia Universitaria, 14(1). DOI: https://doi.org/10.19083/ridu.2020.1174

Moreira, F. S. M., García, B. R. A., Macías, B. A. M. y Vera, J. C. A. (2014). Estrategias para la implementación de un enfoque metodológico interactivo en aulas invertidas para la formación de grado en Educación. Revista Educación y Tecnología, 5, 36-48. Recuperado de: https://dialnet.unirioja.es/servlet/articulo?codigo=5072161

Moreno-Correa, S. M. (2020). La innovación educativa en los tiempos del coronavirus. Salutem Scientia Spiritus, 6(1), 14-26. Recuperado de: https://revistas.javerianacali.edu.co/index.php/salutemscientiaspiritus/article/view/2290

Pascuas, Y. S., Jaramillo, C. O. y Verástegui, F. A. (2015). Desarrollo de objetos virtuales de aprendizaje como estrategia para fomentar la permanencia estudiantil en la educación superior. Revista EAN, 79, 116-129. DOI: https://doi.org/10.21158/01208160.n79.2015.1271

Prensky,M.(2001).Digitalnatives,digitalimmigrants.OntheHorizon,9(5).DOI:https://doi.org/10.1108/10748120110424816

Quijano, Y. (2010). Impacto del uso de entornos virtuales de aprendizaje para la enseñanza de neuroanatomía en estudiantes de medicina. Revista UDCA, 13(2), 15-22. DOI: https://doi.org/10.31910/rudca.v13.n2.2010.724

Roa, D. M. O., Cobo, E. M. y Gómez, G. D. I. (2020). Percepción de los estudiantes de segundo semestre de la carrera de Medicina de la Pontificia Universidad Javeriana (Cali) ante la transición de una modalidad presencial a 
una apoyada en medios digitales durante el tiempo de la pandemia por covid-19. Universitas Médica, 61(4). Recuperado de: https://revistas.javeriana.edu.co/index.php/vnimedica/article/view/30021

Rodrigues, R., Flores, A. C., Peña, T., Castro, G. y Reyes, G. (2009). Uso de Internet del estudiantado de la Universidad Centroamericana, con enfoque en las redes sociales. Encuentro, 84, 44-61. DOI: https://doi.org/10.5377/ encuentro.v41i84.49

Rose, S. (2020). Medical student education in the time of covid-19. JAMA, 323(21), 2131-2132. DOI: https://doi.org/10.1001/ jama.2020.5227

Stanislavski, C. (2016). Ética y disciplina: método de acciones físicas. Moscú: Escenología.

Tinoco-García, A., Osorio, A. y González, F. (2019). Jóvenes, contextos de violencia estructural y ciudadanía. Ultima Década, 27(51), 69-95. DOI: https://doi.org/10.4067/S0718-22362019000100069

Tune, J. D., Sturek, M. y Basile, D. P. (2013). Flipped classroom model improves graduate student performance in cardiovascular, respiratory, and renal physiology. Advances in Physiology Education, 37(4), 316-320. DOI: https://doi.org/10.1152/advan.00o91.2013

Turney, B. W. (2007). Anatomy in a modern medical curriculum. The Annals of The Royal College of Surgeons of England, 89(2), 104-107. DOI: https://doi.org/10.1308/003588407X168244

Valencia, H. G., Valencia, A. R. y Gómez, G. D. I. (2021). El profesor de inglés en los tiempos de uso de la tecnología. Revista Boletín Redipe, 10(3), 317-330. Recuperado de: https://revista.redipe.org/index.php/1/article/ view/1237

Vidal, M., Cañizares, O., Sarasa, N. y Santana, A. (2004). Las nuevas tecnologías en la enseñanza y el aprendizaje de la Anatomía Humana. Educación Médica Superior, 18(4), 1-1. Recuperado de: http://scielo.sld.cu/scielo. php?script=sci_arttext\&pid=So864-21412004000400010

Villatoro, P.y Silva, A. (2005). Estrategias, programas y experiencias de superación de la brecha digital y universalización del acceso a las nuevas tecnologías de información y comunicación (TIC). Un panorama regional. Santiago de Chile: Cepal-Naciones Unidas.

Wodak, R. y Meyer, M. (2003). Métodos de análisis crítico del discurso. España: Gedisa. 